\title{
Introduction to special focus section on prostate cancer imaging
}

\author{
Adam T. Froemming, ${ }^{1}$ Alvin C. Silva ${ }^{2}$ \\ ${ }^{1}$ Department of Radiology, Mayo Clinic, 200 First Street, S.W., Rochester, MN 55905, USA \\ ${ }^{2}$ Department of Radiology, Mayo Clinic, Scottsdale, AZ, USA
}

There have been incredible advancements in prostate MRI in recent years, which have initiated a massive transformation of the way patients are diagnosed and managed. The decades-old management paradigm of random/systematic partial gland sampling followed by epidemiologically based nomograms to forecast likelihood of potential outcomes is coming to an end, and we have begun the monumental shift toward management informed by an individual patient's imaging and targeted biopsy results that more accurately reflect their specific disease status. The implications are enormous, and not just for individual patients that are now able to make treatment decisions based on more reliable data about their disease. Just as importantly, the implications for the benefits for our health care system are equally great, including reduced unnecessary biopsies and costly surgeries; reduced morbidity; improved quality; and hopefully improved outcomes and cost-efficacy. These advancements have been manifest in all facets of prostate imaging, from an incredible number of technical advancements in our MRI capabilities to just as importantly in our understanding and standardization of exam interpretations as guided by PIRADS version 2. In the span of less than 10 years, prostate MRI has evolved from a boutique academic offering, to a widely available mainstream diagnostic test that has recognized value to both clinicians and patients.

As the official journal of the Society of Abdominal Radiology (SAR), this special issue has been assembled under the direction of the SAR's Disease Focused Panel (DFP) on Prostate. With this diagnostic application becoming more widespread, there is great need for practicing radiologists to develop and advance their understanding of prostate imaging. While this Special Issue cannot be comprehensive of such a vast and rapidly evolving landscape, the intent is to serve as a unifying educational source and reference for radiologists, as well as a compliment to other sources such as the excellent ACR PIRADSv2 document and various hands-on workshops.

In this Special Issue, there are a range of review articles spanning from technical considerations in performance of prostate MRI, through step-wise focused attention to each of the individual components of a multiparametric prostate MRI exam. There are also supporting reviews on related aspects of prostate imaging, such as exciting recent advancements in nuclear medicine capabilities; imaging's application to the common clinical scenario of prostate cancer recurrence after definitive management; the interventional side of the practice with targeted biopsy and image-guided focal therapies; and a manuscript offering the perspective and insight of a Urologist and his radiologist colleagues on the impact and future potential of Radiology in this clinical arena. In addition, a selection of Original Research articles has been included.

Finally, the Prostate DFP has identified several key issues that our specialty will need to address in the near future. Short opinion papers from teams of DFP members are included in this special focus edition with the intention of spreading recognition about these issues, and to foster discussion within our community as we collectively work toward solutions. 